\title{
激光双线激发 $\mathrm{La}(\mathbf{I})$ 荧光研究受激粒子的 碰撞能量转移
}

段忆翔*、金巨广 王松岳黄 本 立
(中国科学院长春应用化学研究所) (原门大学化学系)

\section{关链词激光、荧光、铮、能量转移}

通常热助和阶跃苂光 ${ }^{[1]}$ 的产生均是能级上粒子碰撞能量转移的的结果。在某些情况, 这 种粒子碰撞能量转移可以达到相当强烈的程度 ${ }^{[2,3]}$. 基于这一事实, 我们曾经利用粒子碰撞能 量转移所产生的苂光测量能级跃迁的分支比, 并取得了较好的结果 ${ }^{[4]}$. 据我们所知, 有关这种 碰撞能量转移影响因素的系统研究尚未见报道. 本文利用 $\mathrm{La}-\mathrm{Ne}$ 空心阴极灯作为原子化 器, 利用染料激光在 $30 \mathrm{GHz}$ 的扫描范围内首次采用双激发线轮流激发, 研究了影响粒子碰撞 能量转移的一些因素, 从实验上证实了能级差、跃迁几率等因素对粒子碰撞能量转移的影响, 从中总结出一些粒子碰撞能量转移的规律.

\section{一、实验}

实验中用 $\mathrm{La}-\mathrm{Ne}$ 空心阴极灯作为原子化器, 用由昰离子激光器原浦的染料激光器作为 激发源。在染料激光扫描的范围内 $(30 \mathrm{GHz})$, 用 $5930.68 \AA$ 和 $5930.62 \AA$ 作为激发线交替激 发, 同时测量由两条激发线各自产生的苂光. 用三角棱镜在空心阴极灯的顶端收集苂光, 经双

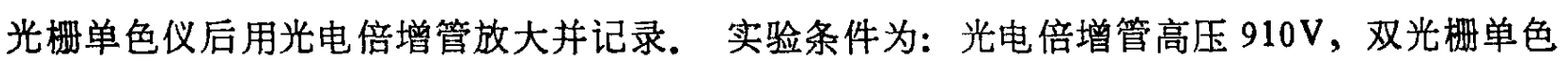
仪狭缝 $100 \mu$, 空心阴极灯电流 $12.5 \mathrm{~mA}$, 激光功率约为 $80 \mathrm{~mW}$. 整个实验装置可参见文献 [4].

\section{二、实验结果与讨论}

1. $\mathrm{La}(\mathrm{I})$ 的能级结构以及两条激发线的比较 根据文献[5-7]中所提供的有关锎的 光谱数据制得 $\mathrm{La}(\mathrm{I})$ 的能级图(图 1). 图中只包括了本实验有意义的部分能级. 实验中我们选 择 $a^{2} D 3 / 2 \rightarrow y^{2} F^{0} 5 / 2\left(5930.68 \AA\right.$ ) 和 $a^{2} D 5 / 2 \rightarrow y^{2} F^{0} 7 / 2$ (5930.62 $\AA$ ) 跃迁作为激发线, 其波 长差为 $0.06 \AA$, 在通常的情况, 一般的光谱方法难以将其分开. 我们利用光电流光谱对两条激 发线进行了分辨并做了谱线强度比较. 所得的光电流信号表明(图 2b), 两条激发线的强度基 本相近. 图 $2 \mathrm{a}$ 中给出了与激发线对应的 $z^{4} G^{0} 7 / 2 \rightarrow a^{2} D 5 / 2$ 跃迁的苂光谱线.

2. 不同的激发线对粒子碰撞能转移的影响按照量子跃迁的选择定则, 我们对图 1 中所给能级跃迁几率较大的谱线进行了测量, 将不同激发线测得的结果列于表 1 中. 为了便 于比较, 我们将表 1 中的数据分为三部分进行讨论. 第一部分只包括 $6455.99 \AA$ 一条谱线, 对 于两条激发线来说, 该线为阶跃荻光线. 由于该谱线的上能级 $\left(z^{2} F^{0} 7 / 2\right)$ 距第一激发线

本文 1988 年千月 2 日收到。1988 年 6 月 24 日收到修改稿.

*吉林大学化学系. 


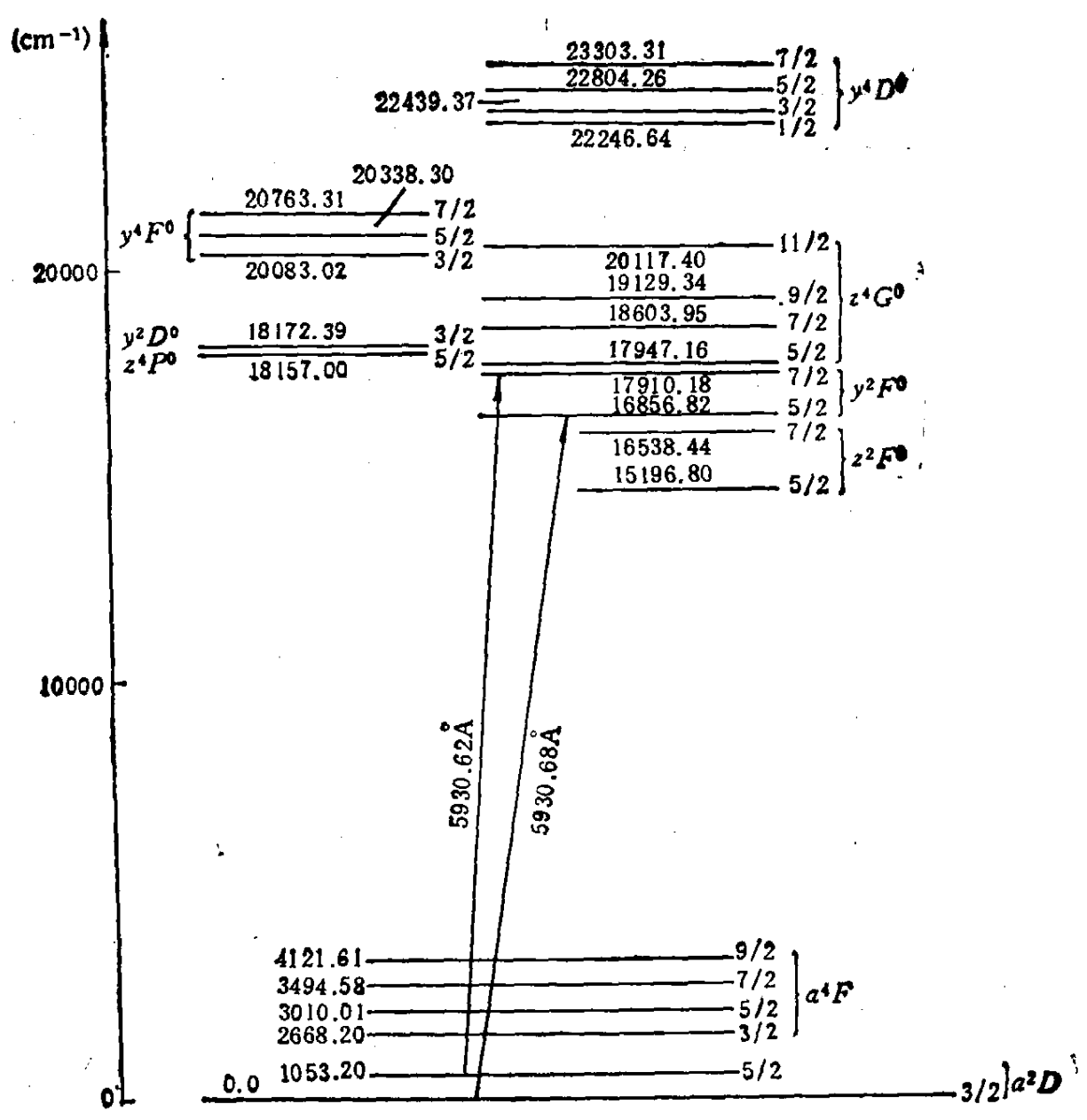

图 $1 \mathrm{La}(\mathrm{I})$ 的部分能级
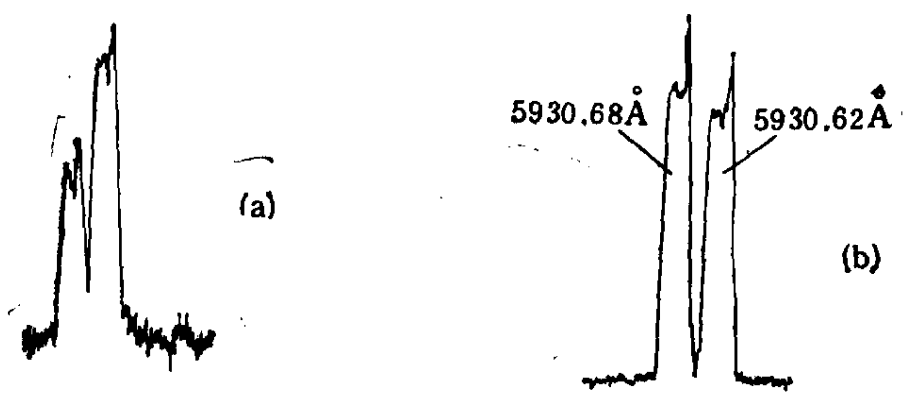

图 2 (a) 与两条激发线对应的荧光光㬐, 跃迁为: $z^{4} G^{0} 7 / 2-a^{2} D 5 / 2$;

(b) 两条数发线的光电流谱

(5930.68 $\AA$ ) 的上能级 $\left(y^{2} F^{0} 5 / 2\right)$ 较近, 而距第二激发线 $(5930.62 \AA)$ 的上能级 $\left(y^{2} F^{0} 7 / 2\right)$ 相对较远，因而由第一激发线泵浦到 $y^{2} F^{0} 5 / 2$ 能级上的粒子较之由第二激发线㫤浦到 $y^{2} F^{0} 7 / 2$ 能级上的粒子更易向 $z^{2} F^{0} 7 / 2$ 能级转移, 所以, 由第一激发线产生的碰撞诱导荧光远大于第

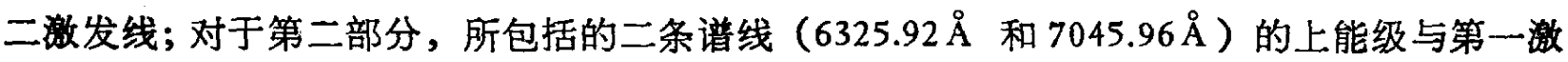
发线的上能级是同一能级,因此, 这二条荧光线对于第一激发线来说是直跃线荧光，不需要碰 撞能量转移,而相对于第二激发线来说,这二条线是阶跃线, 因而需要碰撞能量转移过程, 所 以,来自第一激发线的苂光比来自第二激发线的荧光强; 此后为第三部分，这部分谱线的上能 
表 $1 \mathrm{La}(\mathrm{I})$ 的碰撞能量转移谱线

\begin{tabular}{|c|c|c|c|c|c|c|}
\hline \multirow{2}{*}{ 分 组 } & \multicolumn{2}{|r|}{ 迁 } & \multirow{2}{*}{$\begin{array}{c}\text { 荧光波长 } \\
(\AA)\end{array}$} & \multirow{2}{*}{$\begin{array}{c}\text { 氏迁几事* } \\
\text { (BA) }\end{array}$} & \multicolumn{2}{|c|}{ 苂光强度 } \\
\hline & 上能级 & 下 能 级 & & & $\begin{array}{r}\text { 发线 (1) } \\
(5930.68 \AA \AA\end{array}$ & $\begin{array}{l}\text { 激发线 (II) } \\
(5930.62 \AA)\end{array}$ \\
\hline I & $x^{2} F^{0} 7 / 2$ & $a^{2} D 5 / 2$ & 6455.99 & 0.12 & 789.3 & 92.9 \\
\hline II & $y^{2} F^{0} 5 / 2$ & $\begin{array}{l}a^{2} D 5 / 2 \\
a^{4} F 3 / 2\end{array}$ & $\begin{array}{l}6325.92 \\
7045.96\end{array}$ & $\begin{array}{l}0.056 \\
0.078\end{array}$ & $\begin{array}{l}469.1 \\
331.3\end{array}$ & $\begin{array}{l}12.3 \\
11.8\end{array}$ \\
\hline III & $\begin{array}{l}y^{2} F^{0} 7 / 2 \\
z^{4} G^{0} 5 / 2 \\
z^{4} P^{0} 5 / 2 \\
y^{2} D^{0} 3 / 2 \\
z^{4} G^{0} 7 / 2\end{array}$ & $\begin{array}{l}a^{4} F 5 / 2 \\
a^{2} D 5 / 2 \\
a^{4} F 3 / 2 \\
a^{4} F 5 / 2 \\
a^{2} D 3 / 2 \\
a^{4} F 3 / 2 \\
a^{4} F 5 / 2 \\
a^{2} D 3 / 2 \\
a^{2} D 5 / 2 \\
a^{4} F 5 / 2 \\
a^{4} F 7 / 2 \\
a^{4} F 7 / 2 \\
a^{2} D 5 / 2 \\
a^{4} F 3 / 2 \\
a^{4} F 5 / 2 \\
a^{4} F 5 / 2 \\
a^{4} F 3 / 2 \\
a^{4} F 5 / 2 \\
a^{4} F 5 / 2 \\
a^{4} F 7 / 2\end{array}$ & $\begin{array}{l}6709.50 \\
5917.63 \\
6543.16 \\
6692.87 \\
5506.00 \\
6454.52 \\
6600.17 \\
5501.34 \\
5696.19 \\
6410.99 \\
6616.59 \\
6394.23 \\
5183.92 \\
5657.72 \\
5769.34 \\
5631.22 \\
5056.46 \\
5145.42 \\
5050.57 \\
5177.31\end{array}$ & $\begin{array}{l}0.15 \\
0.032 \\
0.10 \\
0.036 \\
0.036 \\
0.073 \\
0.027 \\
0.32 \\
0.073 \\
0.19 \\
0.049 \\
0.46 \\
0.11 \\
0.17 \\
0.48 \\
0.23 \\
0.36 \\
0.95 \\
0.49 \\
1.4\end{array}$ & $\begin{array}{r}39.3 \\
4.6 \\
25.2 \\
1.7 \\
0.3 \\
12.3 \\
0.5 \\
1.7 \\
5.1 \\
21.1 \\
5.4 \\
12.1 \\
2.1 \\
3.0 \\
15.2 \\
6.7 \\
1.7 \\
6.2 \\
1.7 \\
10.3\end{array}$ & $\begin{array}{r}1000 \\
47.8 \\
252.3 \\
34.1 \\
1.0 \\
29.1 \\
12.8 \\
14.4 \\
6.7 \\
26.3 \\
5.9 \\
13.3 \\
2.3 \\
2.8 \\
14.3 \\
6.7 \\
2.2 \\
7.9 \\
1.7 \\
10.3\end{array}$ \\
\hline
\end{tabular}

* 引自文献 [8].

级距离第二激发线的上能级较近，因而受第二激发线的影响较第一激发线为大,来自第二激发 线上能级 $\left(y^{2} F^{07} / 2\right)$ 的粒子碰撞能量转移要较第一激发线容易, 所以, 实验中第二激发线给 出较强的苂光,而且,距第二激发线的上能级越近，也即热助距离越短，由第二激发线所获得的 苂光强度越大.

值得注意的是, 在第三部分数据中, 当第二激发线上能级的热助高度 (粒子碰撞能量转移 的距离)超过二条激发线上能级之间差值 $\left(1053.36 \mathrm{~cm}^{-1}\right)$ 约一倍以上时,两条激发线对苂光强 度的影响差异不再明显, 出现拉平的趋势. 这一实验结果也表明粒子的碰撞能量转移超出一 定的距离后, 能级差的影响将大大减小, 只有在一定的范围内, 能级差对粒子碰撞能量转移起 主导作用.

3. 同一做发线的定五比较为了进一步阐明能级差对粒子碰撞能量转移的影响, 我们 对实验中所遇到的几组跃迁几率相同, 能级差不同的谱线进行了定量比较。用第二激发线激 发,所得结果列人表 2 中. 由表 2 可见, 在跃迁几率相同的条件下,随着能级差的增大, 粒子碰 撞能量转移的强度下降。

4. 荻光线跃迁几事对柆子碰接能五转移的影响 为了考察荧光线跃迁几率这一因素对 
表 2 相同跃迁几率,不同能级 差的荧光谱线强度的比较

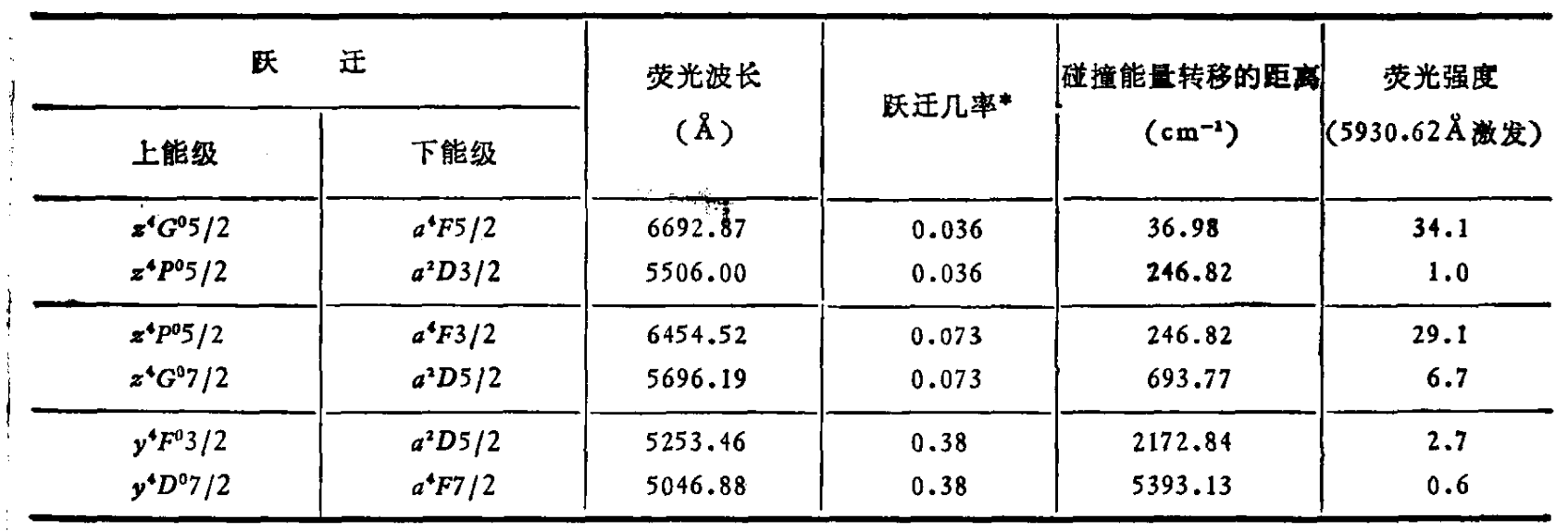

*引自文献 [8].

粒子碰撞能量转移的影响，我们在双光栅单色仪的允许波长范围内,选用第二激发线激发,测 量了 $y^{4} F^{0} 3 / 2$ 和 $z^{4} G^{0} 11 / 2$ 能级的全部允许跃迁，并进行了总强度的比较. 鉴于 $y^{4} F^{0} 3 / 2$ 和 $z^{4} G^{0} 11 / 2$ 能级只相差 $34.38 \mathrm{~cm}^{-1}$ ，且远离受激上能级，因此我们有理由忽略 $y^{4} F^{0} 3 / 2$ 和 $\boldsymbol{z}^{4} G^{0} 11 / 2$ 之间能级差的影响. 考虑到粒子的碰撞能量转移应该处于动态平衡过程, 高能级上 的粒子通过各种途径跃迁回到基态或低激发态,若这种去激发途径的跃迁几率较大,则粒子跃 迁回到基态或低激发态的数目较多,为了维持动态平衡,需要由受激上能级转移到该跃迁上能 级的粒子数也较多,因此,去激发途径的跃迁几率越大，通常该跃迁上能级所获得的能量转栘 越强烈，也即跃迁几率在某种程度上对粒子碰撞能量转移有疏导作用，我们的实验结果证明了 这一点.

\section{三、结 论}

从本文的实验结果,我们初步得到如下结论:

1. 在距离受激上能级较近的一定能级范围内, 能级差对粒子碰撞能量转移起主导作用,距 受激上能级越近,能级差的影响越明显.

2. 超出一定的能级差范围 (本实验中约为 $1000 \mathrm{~cm}^{-1}$ ), 能级差对粒子碰撞能量转移的影响 减小。不同激发线所产生的茨光强度趋于拉平.

3. 苂光谱线的跃迁几率对粒子碰撞能量转移有疏导作用.

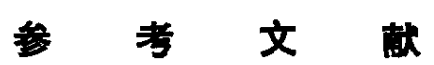

[1] Omenetto, N. and Winefordner, J. D., Appl. Spectrosc., 26(1972), 555.

[2] 段䎲翔、金巨广、黄本立,分析化学，16(1988),1080.

[3] 段忆联、王松岳、金巨广、黄本立，应用化学，5(1988),5: 74.

[4] 金巨广、段䎲翔、王松岳、黄本立,科学通报, 33(1988), 8: 588 .

[5]冶金工业部科技情报研究所编译,光谱线波长表,中国工业出版社，1971。

[6] Moore, C. E., Atomic energy level, NBS Monograph 35, Washington D. C., 1971.

[7] Harrison, G. R., Rosin, N. and Mcnally, Jr. J. R., J. Opti. Soci. Amer., 35(1945), 658.

[8] Corliss, C. H. and Bozman, W. R., Experimental transition probabilities, NBS Monograph 53, Washington D. C. 1962. 